\title{
The Factors Reducing Relative Dose Intensity of Cisplatin and Vinorelbine as Adjuvant Chemotherapy in Patients with Non-Small Cell Lung Cancer
}

\author{
Ryo Inose ${ }^{1}, K^{2}$ atsuyuki Takahashi ${ }^{1 凶}$, Naruo Yoshimura², Tomoya Kawaguchi², Mitsutaka Takada ${ }^{3}$, Katsuya \\ Nagayama $^{1}$ \\ 1. Department of Pharmacy, Osaka City University Hospital, 1-5-7, Asahi-machi, Abeno-ku, Osaka-shi, Osaka, 545-8586, Japan; \\ 2. Department of Respiratory Medicine, Graduate School of Medicine, Osaka City University, 1-4-3, Asahi-machi, Abeno-ku, Osaka \\ 545-8585, Japan; \\ 3. Division of Clinical Drug Informatics, School of Pharmacy, Kinki University, 3-4-1, Kowakae, Higashiosaka-shi, Osaka, 577-0818, Japan.
}

$\square$ Corresponding author: Katsuyuki Takahashi, Ph.D, Department of Pharmacy, Osaka City University Hospital, 1-5-7, Asahi-machi, Abeno-ku, Osaka-shi, Osaka, 545-8586, Japan. Telephone: +81-6-6645-2121; Fax: +81-6-6646-1734; E-mail: k.taka@med.osaka-cu.ac.jp

(c) Ivyspring International Publisher. This is an open access article distributed under the terms of the Creative Commons Attribution (CC BY-NC) license (https://creativecommons.org/licenses/by-nc/4.0/). See http://ivyspring.com/terms for full terms and conditions.

Received: 2017.06.02; Accepted: 2017.07.28; Published: 2018.01.01

\begin{abstract}
Purpose Adjuvant therapy with cisplatin and vinorelbine (CV therapy) is recommended for patients after complete surgical resection of stage II and IIIA non-small cell lung cancer (NSCLC). However, a dose reduction or early discontinuation of a subsequent course of treatment is a frequent clinical problem. The aim of this study was to investigate the factors reducing the relative dose intensity (RDI) of adjuvant $\mathbf{C V}$ therapy in patients with NSCLC.

Methods Patients who had received CV as an adjuvant chemotherapy for NSCLC at the Osaka City University Hospital between July 2007 and March 2016 were identified from medical records. The patients were classified into two groups (RDI $<50 \%$ and $\mathrm{RDI} \geq 50 \%$, respectively), and patients' characteristics, laboratory values, adverse reactions, and RDI of CV therapy were analyzed. The two groups were compared using the Fisher's exact test.

Results The study population consisted of 25 patients. There were six patients (24.0\%) with RDI $<50 \%$ and 19 patients $(76.0 \%)$ with $\mathrm{RDI} \geq 50 \%$. Univariate analysis showed that the use of calcium channel blockers [crude odd ratio (OR): 10.70; 95\% confidence interval (Cl): 1.31-86.94; $p=0.032$ ] and renal dysfunction (creatinine clearance $<60 \mathrm{~mL} / \mathrm{min}$ ) (crude OR: 18.00; $95 \% \mathrm{Cl}: 1.37-235.70$; $p=0.031$ ) were associated with the reduced RDI.

Conclusion This study indicated that the renal dysfunction and concomitant use of calcium channel blockers might be predictive factors for reduced RDI of adjuvant CV therapy in patients with NSCLC. Patients with these factors may be able to avoid extreme reduction in RDI if the dose is reduced or the dosing interval is prolonged.
\end{abstract}

Key words: adjuvant therapy, cisplatin, vinorelbine, non-small cell lung cancer.

\section{Introduction}

Lung cancer is one of the most common cancers and a major public health problem. Lung cancer related deaths are highly prevalent worldwide, with lung cancer being the leading cause of cancer mortality in men and the second leading cause of cancer mortality in women. [1]. Lang cancer is categorized as small and non-small cell lung cancer (NSCLC) (about 10\% and 90\% of cases in Japan, 
respectively) [2]. Surgical resection is the standard therapy for patients with completely resectable NSCLC. Although operable NSCLC is totally removed by surgery, patients frequently develop a local and distant recurrence, leading to death, and a distant recurrence occurs more frequently than local recurrence at any stage [3]. The cause of recurrence is thought to be micrometastatic lesions. Therefore, adjuvant chemotherapy is needed for patients with NSCLC to prevent a relapse. A number of clinical trials have been conducted on adjuvant chemotherapy for patients with NSCLC to reduce the recurrence rate and prolong overall survival [4-8]. A meta-analysis of five trials that evaluated the effectiveness of adjuvant chemotherapy has reported that postoperative cisplatin-based chemotherapy significantly improved survival in patients with NSCLC [9]. Subgroup analysis of cisplatin (CDDP) and vinorelbine (VNB) therapy (CV therapy) found no clinical benefits for patients with stage I NSCLC [10]. On the other hand, the 5-year overall survival of patients with stages II and IIIA significantly improved, by $11.6 \%$ and $14.7 \%$, respectively. Based on these results, adjuvant $\mathrm{CV}$ therapy is recommended for patients after complete surgical resection of stage II and IIIA NSCLC.

It is generally considered that keeping the relative dose intensity (RDI) high is important in adjuvant chemotherapy [11-12]. For instance, in adjuvant chemotherapy with cyclophosphamide, methotrexate, and fluorouracil for breast cancer, maintaining RDI $\geq 85 \%$ is recommended to prolong the relapse-free survival and overall survival [11]. Likewise, in colon cancer, RDI of adjuvant chemotherapy is known to affect the prognosis [12]. As with breast and colon cancer, keeping high RDI is expected to improve the prognosis in NSCLC patients treated with adjuvant CV therapy. However, a dose reduction or discontinuation of a subsequent course after initial treatment frequently becomes a clinical problem for patients treated with $\mathrm{CV}$ therapy. Meanwhile, factors reducing RDI of adjuvant $\mathrm{CV}$ therapy have not been identified. Therefore, identification of the factors reducing RDI of adjuvant $\mathrm{CV}$ therapy may be beneficial in terms of improving the prognosis, as with breast and colon cancer. The aim of this study was to investigate the factors reducing $\mathrm{RDI}$ of adjuvant $\mathrm{CV}$ therapy in patients with NSCLC.

\section{Methods}

\section{Study patients}

Patients who had received CV therapy as adjuvant chemotherapy for NSCLC at the Osaka City
University Hospital between July 2007 and March 2016 were identified from medical records. Patients who discontinued the therapy because of their wishes or for unknown reasons, those who participated in clinical trials relevant to $\mathrm{CV}$ therapy, and patients with progressive disease during $\mathrm{CV}$ therapy were excluded from the study. The included patients were classified into two groups (RDI $<50 \%$ and RDI $\geq 50 \%$, respectively). Adjuvant $\mathrm{CV}$ therapy consisted of CDDP $\left(80 \mathrm{mg} / \mathrm{m}^{2}\right.$, day 1$)$ and $\operatorname{VNB}\left(25 \mathrm{mg} / \mathrm{m}^{2}\right.$, days 1 and 8) administered every 3 weeks for four cycles. When Grade 3 or higher non-hematologic toxicity or Grade 4 hematologic toxicity appeared, the doses of CDDP and VNB in the subsequent cycles were reduced to 60 and $20 \mathrm{mg} / \mathrm{m}^{2}$, respectively. Otherwise, dose reduction or discontinuation of the $\mathrm{CV}$ therapy was based on the physician's discretion. This study was approved by the institutional ethics committee (No. 3576) on September 30, 2016.

\section{Assessment}

Patients' characteristics (sex, age, body mass index, histologic type, disease stage, lymph nodal status, time between surgery and CV therapy, and combined drugs), laboratory values, and adverse reactions were analyzed. RDI of $\mathrm{CV}$ therapy was determined as the average of RDI values for CDDP and VNB. Adverse reactions were evaluated using the Common Terminology Criteria for Adverse Events ver. 4.0. We defined adverse reactions of grade $\geq 3$ as serious adverse reactions.

\section{Statistical analysis}

The two groups were compared using the Fisher's exact test. $P$-values were reported as two-sided, and values of less than 0.05 were considered statistically significant. IBM SPSS ${ }^{\circledR}$ Statistics 22.0 (SPSS Japan, Inc., Tokyo, Japan) was used for all statistical analyses.

\section{Results}

\section{Patients' characteristics}

Fig.1 shows the flowchart for study inclusion. There were 39 patients who received CV therapy during the study period. Of these, the following 14 patients were excluded from the study: 1) patients who discontinued the therapy on their wishes (four patients) and for unclear reasons (three patients); 2) five patients who participated in clinical trials relevant to $\mathrm{CV}$ therapy; and 3) two patients with progressive disease during $\mathrm{CV}$ therapy. Consequently, 25 patients were included in the study, and their data were analyzed. The detailed 
characteristics of these 25 patients are shown in Table 1 , of which $28 \%(7 / 25)$ were treated with calcium channel blockers during CV therapy. The types of calcium channel blockers used were amlodipine $(\mathrm{n}=4)$, nifedipine $(\mathrm{n}=2)$, and verapamil $(\mathrm{n}=1)$.

\section{Toxicities}

The adverse reactions observed in the study are shown in Table 2. The data showed that all patients experienced some adverse reactions. Serious leukopenia and neutropenia occurred in 21 (84.0\%) and $24(96.0 \%)$ patients, respectively. Nine patients $(36.0 \%)$ experienced febrile neutropenia. Serious anemia occurred in two patients (8.0\%). Serious elevations of aspartate aminotransferase and creatinine were observed in one patient $(4.0 \%)$, and alanine aminotransferase was seriously elevated in another patient $(4.0 \%)$. No treatment-related deaths were recorded in this study.

\section{Distribution of RDI}

The mean RDI values were $66.0 \%$ and $59.0 \%$ for CDDP and VNB, respectively, and the median RDIs were $73.6 \%$ (range: $25.0-100.0$ ) and $60.4 \%$ (range: 12.5-93.0) for CDDP and VNB, respectively. The overall median RDI was $68.8 \%$ (range: $18.8-93.8$ ) for $\mathrm{CV}$ therapy. The distribution of RDI values in the study patients is presented in Fig. 2. There were six patients $(24.0 \%)$ with RDI $<50 \%$ and 19 patients (76.0\%) with RDI $\geq 50 \%$. Discontinuation of $\mathrm{CV}$ therapy by the patients with RDI $<50 \%$ was attributable to renal dysfunction.
Table 1. Characteristics of the study patients

\begin{tabular}{ll}
\hline Gender (male/female) & $8 / 17$ \\
\hline Age* $^{*}$ & $65(44-75)$ \\
BMI $^{*}$ & $21.8(16.1-27.9)$ \\
Histologic type & \\
Squamous & 6 \\
Non-Squamous & 19 \\
Stage (I/II/III/IV) & $0 / 11 / 14 / 0$ \\
Time from surgery to chemotherapy (days) & $49(29-104)$ \\
$\begin{array}{l}\text { Combination with calcium channel blocker } \\
\text { (yes/no) }\end{array}$ & $7 / 18$
\end{tabular}

BMI: Body mass index

*: Median (range)

Table 2. Toxicities

\begin{tabular}{lll}
\hline Adverse reaction & Any grade $(\%)$ & grade $\geq 3(\%)$ \\
\hline Leukopenia & $25(100.0)$ & $21(84.0)$ \\
Neutropenia & $25(100.0)$ & $24(96.0)$ \\
Anemia & $24(96.0)$ & $2(8.0)$ \\
Thrombocytopenia & $21(84.0)$ & $0(0)$ \\
AST increased & $12(48.0)$ & $1(4.0)$ \\
ALT increased & $13(52.0)$ & $1(4.0)$ \\
Total bilirubin increased & $7(28.0)$ & $0(0)$ \\
Creatinine increased & $11(44.0)$ & $1(4.0)$ \\
Hypoalbuminemia & $11(44.0)$ & $0(0)$ \\
Nausea & $14(56.0)$ & $5(20.0)$ \\
Vomiting & $3(12.0)$ & $2(8.0)$ \\
\hline
\end{tabular}

AST: Aspartate aminotransferase

ALT: Alanine aminotransferase

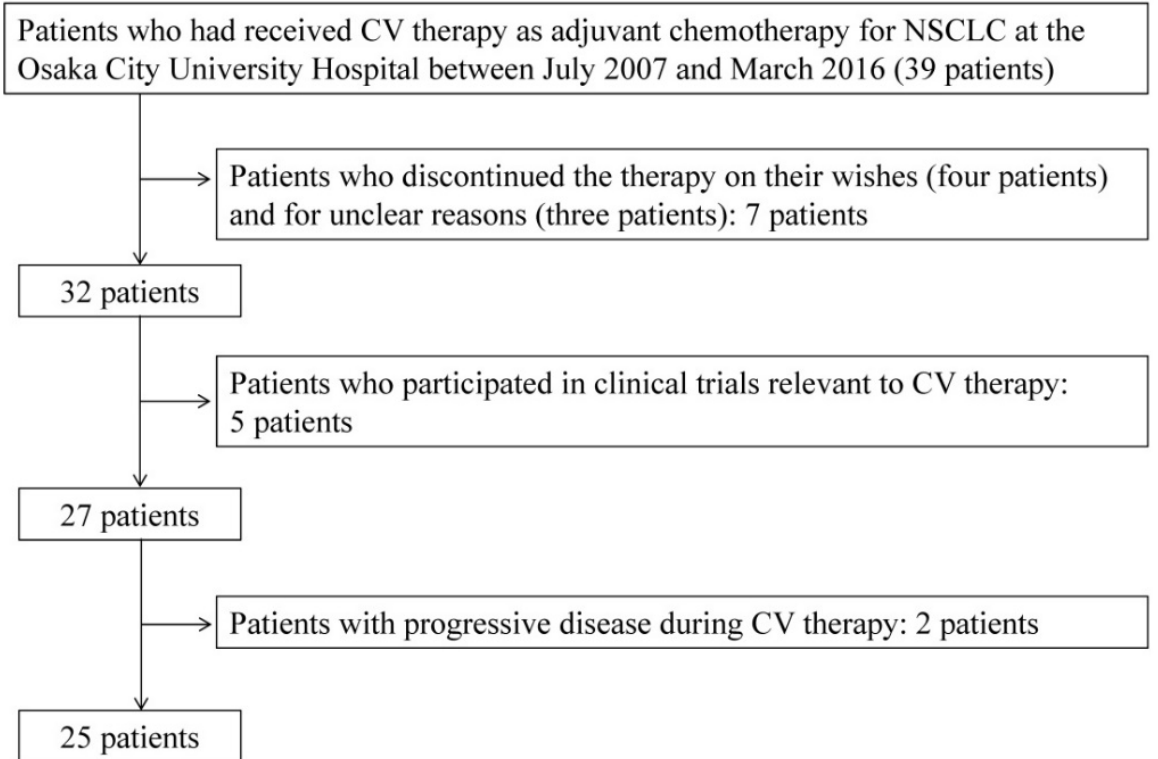

Figure 1. Flowchart of patient selection 
Table 3. Univariate analysis of the factors reducing RDI (patients' background data and laboratory values before starting chemotherapy)

\begin{tabular}{|c|c|c|c|c|c|}
\hline & \multicolumn{2}{|l|}{ RDI } & \multirow{2}{*}{$\begin{array}{l}\text { Odds } \\
\text { ratio }\end{array}$} & \multirow{2}{*}{$\begin{array}{l}95 \% \\
\text { confidence } \\
\text { interval }\end{array}$} & \multirow[t]{2}{*}{ p-value } \\
\hline & $<50 \%$ & $\geq 50 \%$ & & & \\
\hline \multicolumn{6}{|l|}{ Gender } \\
\hline Male & $4(66.7)$ & $13(68.4)$ & 0.92 & $0.13-6.51$ & 0.65 \\
\hline Female & $2(33.3)$ & $6(31.6)$ & & & \\
\hline \multicolumn{6}{|l|}{ Age } \\
\hline$\geq 65$ & $3(50.0)$ & $11(57.9)$ & 0.73 & $0.12-4.59$ & 0.55 \\
\hline$<65$ & $3(50.0)$ & $8(42.1)$ & & & \\
\hline \multicolumn{6}{|l|}{ BMI } \\
\hline$<18.5$ & $1(16.7)$ & $3(15.8)$ & 1.07 & $0.090-12.69$ & 0.69 \\
\hline$\geq 18.5$ & $5(83.3)$ & $16(84.2)$ & & & \\
\hline \multicolumn{6}{|l|}{ Histologic type } \\
\hline Squamous & $1(16.7)$ & $5(26.3)$ & 0.56 & $0.052-6.04$ & 0.55 \\
\hline Non-squamous & $5(83.3)$ & $14(73.7)$ & & & \\
\hline \multicolumn{6}{|l|}{ Stage } \\
\hline III & $2(33.3)$ & $12(63.2)$ & 0.29 & $0.042-2.02$ & 0.21 \\
\hline II & $4(66.7)$ & $7(36.8)$ & & & \\
\hline \multicolumn{6}{|c|}{ Lymph nodal status } \\
\hline$\geq \mathrm{N} 1$ & $4(66.7)$ & $16(84.2)$ & 0.38 & $0.046-3.06$ & 0.34 \\
\hline No & $2(33.3)$ & $3(15.8)$ & & & \\
\hline \multicolumn{6}{|c|}{ Time from surgery to chemotherapy (days) } \\
\hline$\geq 50$ & $3(50.0)$ & $9(47.4)$ & 1.11 & $0.18-6.97$ & 0.64 \\
\hline$<50$ & $3(50.0)$ & $10(52.6)$ & & & \\
\hline \multicolumn{6}{|c|}{ Combination with calcium channel blocker } \\
\hline Yes & $4(66.7)$ & $3(15.8)$ & 10.70 & $1.31-86.94$ & 0.032 \\
\hline No & $2(33.3)$ & $16(84.2)$ & & & \\
\hline \multicolumn{6}{|l|}{ Leukopenia } \\
\hline Grade 0 & $6(100.0)$ & $19(100.0)$ & - & - & - \\
\hline Grade $\geq 1$ & $0(0)$ & $0(0)$ & & & \\
\hline \multicolumn{6}{|l|}{ Neutropenia } \\
\hline Grade 0 & $6(100.0)$ & $19(100.0)$ & - & - & - \\
\hline Grade $\geq 1$ & $0(0)$ & $0(0)$ & & & \\
\hline \multicolumn{6}{|l|}{ Anemia } \\
\hline Grade 0 & $4(66.7)$ & $16(84.2)$ & 0.38 & $0.046-3.06$ & 0.34 \\
\hline Grade $\geq 1$ & $2(33.3)$ & $3(15.8)$ & & & \\
\hline \multicolumn{6}{|c|}{ Thrombocytopenia } \\
\hline Grade 0 & $6(100.0)$ & 15 (78.9) & - & - & 0.31 \\
\hline Grade $\geq 1$ & $0(0)$ & $4(21.1)$ & & & \\
\hline \multicolumn{6}{|l|}{ AST increased } \\
\hline Grade 0 & $6(100.0)$ & $16(84.2)$ & - & - & 0.42 \\
\hline Grade $\geq 1$ & $0(0)$ & $3(15.8)$ & & & \\
\hline \multicolumn{6}{|l|}{ ALT increased } \\
\hline Grade 0 & $6(100.0)$ & $18(94.7)$ & - & - & 0.76 \\
\hline Grade $\geq 1$ & $0(0)$ & $1(5.3)$ & & & \\
\hline \multicolumn{6}{|l|}{ CLcr (ml/min) } \\
\hline $60>$ & $3(50.0)$ & $1(5.3)$ & 18.00 & $1.37-235.70$ & 0.031 \\
\hline $60 \leq$ & $3(50.0)$ & $18(94.7)$ & & & \\
\hline \multicolumn{6}{|c|}{ Hypoalbuminemia } \\
\hline Grade 0 & $6(100.0)$ & $18(94.7)$ & - & - & 0.76 \\
\hline Grade $\geq 1$ & $0(0)$ & $1(5.3)$ & & & \\
\hline
\end{tabular}

RDI: Relative Dose Intensity, AST: Aspartate aminotransferase, CLcr: Creatinine clearance, BMI: Body Mass Index, ALT: Alanine aminotransferase

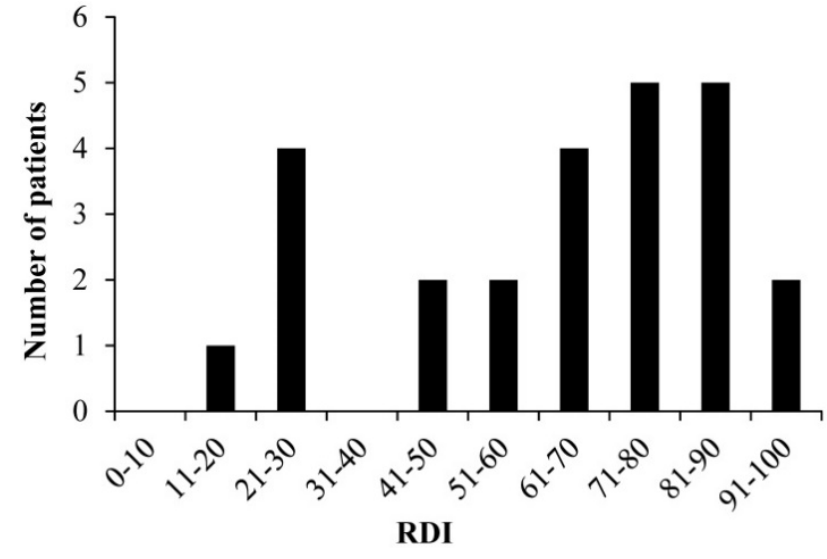

Figure 2. Distribution of RDI RDI: relative dose intensity

\section{Univariate analysis}

Univariate analysis showed that the use of calcium channel blockers [crude odds ratio (OR): 10.70; 95\% confidence interval (CI): 1.31-86.94; $p=0.032$ ] and renal dysfunction [creatinine clearance (CLcr) $<60 \mathrm{~mL} / \mathrm{min}$ ] (crude OR: 18.00; 95\% CI: 1.37-235.70; $p=0.031]$ were associated with reduced RDI (Table 3). No other factors were found to reduce RDI.

\section{Discussion}

This study revealed that the renal dysfunction (CLcr $<60 \mathrm{~mL} / \mathrm{min}$ ) and concomitant use of calcium channel blockers were associated with the decreased RDI of adjuvant CV therapy in patients with NSCLC. Kenmotsu et al. have reported that characteristics of patients, including their gender, age, smoking status, performance status, pathological stage, surgical procedure, and time from surgery to chemotherapy, were not associated with the CV therapy compliance [13]. However, it has not been reported whether there was an association with renal function and the concomitant use of drugs.

Adjuvant CV therapy consists of four cycles of administration every 3 weeks. A dose reduction or discontinuation of the subsequent course at an early cycle frequently becomes a clinical problem in patients treated with $\mathrm{CV}$ therapy. We defined the discontinuation at fewer than two cycles as an early discontinuation and RDI $<50 \%$ as the cutoff value.

In our study, the use of calcium channel blockers was associated with reduced RDI $(p=0.032)$. Vinca alkaloids are metabolized by the cytochrome P450 isoform CYP3A4, and their metabolism is inhibited by CYP3A4 inhibitors such as macrolide antibiotics and calcium channel blockers. [14-16] Indeed, it has been reported that clarithromycin increased the risk of neutropenia, which is a dose-limiting toxicity of VNB 
[15]. Likewise, some calcium channel blockers may interact with VNB and increase the possibility of severe neutropenia. However, in our study, all patients with $\mathrm{RDI}<50 \%$ discontinued $\mathrm{CV}$ therapy because of renal dysfunction. VNB is generally considered to cause less risk of renal dysfunction; therefore, the decreased RDI of adjuvant CV therapy might not be associated with the enhancement of VNB toxicity. Accordingly, we focused on the relationship between calcium channel blockers and CDDP. There have been reports suggesting that calcium channel blockers increase or decrease the renal toxicity of CDDP [17-19]. For instance, it was reported that nifedipine increased the renal toxicity of CDDP in rats [17]. On the other hand, it was reported that verapamil prevented the CDDP-induced renal dysfunction [18-19]. Consequently, the drug interactions of CDDP and calcium channel blockers remain unclear. In our study, calcium channel blockers were identified as the potential predictor of RDI $<50 \%$. However, other antihypertensive drugs, including loop diuretics, aldosterone antagonists, angiotensin-converting enzyme inhibitors, and angiotensin II receptor blockers, were not associated with the reduced RDI (data not shown). A further study is needed to clarify the association between reduced RDI and the use of calcium channel blockers in patients treated with CV therapy.

In this study, decreased renal function (CLcr $<60$ $\mathrm{mL} / \mathrm{min}$ ) before the initiation of $\mathrm{CV}$ therapy was also associated with the decreased RDI $(p=0.031)$. Although there were four cases with decreased renal function in the study, their CDDP dose was not reduced in the first course. The reason for this was the criterion for renal dysfunction had been defined in a Phase II study of Japanese patients as a serum creatinine level of $\geq 1.5 \mathrm{mg} / \mathrm{dL}$ [20], but none of the patients from this study met this criterion. Therefore, the patients with renal dysfunction were treated with the full dose of CDDP. Thus, the dose of CDDP should be determined based not only on the serum creatinine level but also on CLcr.

Several potential limitations should be taken into account when interpreting the results obtained in this study. First, this was an observational study with a retrospective design, performed at a single hospital, and the available sample size was small. Therefore, non-hematologic toxicities were underestimated in our study. Second, patients with RDI $<50 \%$ were defined as a low RDI group in this study. It is usually considered that RDI of more than $\sim 85 \%$ is needed for effective adjuvant chemotherapy. In this study, predictive factors for extremely reduced RDI were investigated. Therefore, $50 \%$ was used as the cutoff value to divide the patients into two groups. Despite these limitations, this study suggests that calcium channel blockers and decreased renal function may be associated with reduced RDI of adjuvant CV therapy in patients with NSCLC.

\section{Conclusion}

This study indicated that renal dysfunction and a concomitant use of calcium channel blockers may be predictive factors for reduced RDI of adjuvant CV therapy in patients with NSCLC.

\section{Competing Interests}

The authors have declared that no competing interest exists.

\section{References}

1. Jemal A, Bray F, Center MM, et al. Global cancer statistics. CA: a cancer journal for clinicians. 2011; 61: 69-90.

2. Sawabata N, Asamura H, Goya T, et al. Japanese Lung Cancer Registry Study: first prospective enrollment of a large number of surgical and nonsurgical cases in 2002. J Thorac Oncol. 2010; 5: 1369-75.

3. Pisters KM, Le Chevalier T. Adjuvant chemotherapy in completely resected non-small-cell lung cancer. J Clin Oncol. 2005; 23: 3270-8.

4. Arriagada R, Bergman B, Dunant A, et al. Cisplatin-based adjuvant chemotherapy in patients with completely resected non-small-cell lung cancer. The New England journal of medicine. 2004; 350: 351-60.

5. Winton T, Livingston R, Johnson D, et al. Vinorelbine plus cisplatin vs. observation in resected non-small-cell lung cancer. The New England journal of medicine. 2005; 352: 2589-97.

6. Douillard JY, Rosell R, De Lena M, et al. Adjuvant vinorelbine plus cisplatin versus observation in patients with completely resected stage IB-IIIA non-small-cell lung cancer (Adjuvant Navelbine International Trialist Association [ANITA]): a randomised controlled trial. The Lancet Oncology. 2006; 7: 719-27

7. Scagliotti GV, Fossati R, Torri V, et al. Randomized study of adjuvant chemotherapy for completely resected stage I, II, or IIIA non-small-cell Lung cancer. Journal of the National Cancer Institute. 2003; 95: 1453-61.

8. Waller D, Peake MD, Stephens RJ, et al. Chemotherapy for patients with non-small cell lung cancer: the surgical setting of the Big Lung Trial. Eur J Cardiothorac Surg. 2004; 26: 173-82.

9. Pignon JP, Tribodet H, Scagliotti GV, et al. Lung adjuvant cisplatin evaluation: a pooled analysis by the LACE Collaborative Group. J Clin Oncol. 2008; 26: 3552-9.

10. Douillard JY, Tribodet H, Aubert D, et al. Adjuvant cisplatin and vinorelbine for completely resected non-small cell lung cancer: subgroup analysis of the Lung Adjuvant Cisplatin Evaluation. J Thorac Oncol. 2010; 5 : 220-8

11. Bonadonna G, Valagussa P, Moliterni A, et al. Adjuvant cyclophosphamide, methotrexate, and fluorouracil in node-positive breast cancer: the results of 20 years of follow-up. The New England journal of medicine. 1995; 332: 901-6.

12. Aspinall SL, Good CB, Zhao X, et al. Adjuvant chemotherapy for stage III colon cancer: relative dose intensity and survival among veterans. BMC cancer. 2015; 15: 62

13. Kenmotsu H, Ohde $Y$, Shukuya $T$, et al. Feasibility of postoperative adjuvant chemotherapy of cisplatin plus vinorelbine for completely resected non-small-cell lung cancer: a retrospective study in Japan. Respiratory investigation. 2012; 50: 157-61.

14. Kajita J, Kuwabara T, Kobayashi H, et al. CYP3A4 is mainly responsibile for the metabolism of a new vinca alkaloid, vinorelbine, in human liver microsomes. Drug metabolism and disposition. 2000; 28: 1121-7.

15. Yano R, Tani D, Watanabe K, et al. Evaluation of potential interaction between vinorelbine and clarithromycin. The Annals of pharmacotherapy. 2009; 43: 453-8.

16. Fedeli L, Colozza M, Boschetti E, et al. Pharmacokinetics of vincristine in cancer patients treated with nifedipine. Cancer. 1989; 64: 1805-11.

17. Deray G, Dubois M, Beaufils H, et al. Effects of nifedipine on cisplatinum-induced nephrotoxicity in rats. Clinical nephrology. 1988; 30: $146-50$.

18. Sleijfer DT, Offerman JJ, Mulder NH, et al. The protective potential of the combination of verapamil and cimetidine on cisplatin-induced nephrotoxicity in man. Cancer. 1987; 60: 2823-8. 
19. Offerman JJ, Meijer S, Sleijfer DT, et al. The influence of verapamil on renal function in patients treated with cisplatin. Clinical nephrology. 1985; 24: 249-55.

20. Sonobe M, Okubo K, Teramukai S, et al. Phase II study of adjuvant vinorelbine and cisplatin in Japanese patients with completely resected stage II and III non-small cell lung cancer. Cancer chemotherapy and pharmacology. 2014; 74: 1199-206. 